\title{
A Poincaré-Birkhoff-Witt Theorem for Quantized Universal Enveloping Algebras of Type $A_{N}$
}

By

\author{
Hiroyuki YAMANE*
}

\section{Introduction}

In this paper, we construct an explicit basis of the quantized universal enveloping algebra $U_{q}\left(s l_{N+1}(\mathbf{C})\right)$.

Let $A=\left(a_{i j}\right)_{1 \leq i, j \leq N}$ be a symmetrizable generalized Cartan matrix, and $\mathscr{G}(A)$ the Kac-Moody Lie algebra of $A$. Motivated by studies of quantum YangBaxter equations, Jimbo [6] and Drinfeld $[2,3]$ introduced a Hopf algebra $U_{q}(\mathscr{G}(A))$ with a nonzero complex parameter $q$. This Hopf algebra, which is also called [3] a "quantum group", can be considered as a natural $q$-analogue of the universal enveloping algebra $U(\mathscr{G}(A))$ of $\mathscr{G}(A)$. For example, it is known that the representation theory of $U_{q}(\mathscr{G}(A))$ is quite analogous to that of $U(\mathscr{G}(A))$. See Lusztig [9] and Rosso [11]. The purpose of this paper is to show that, if $\mathscr{G}(A)$ is of type $A_{N}$, and $q^{8} \neq 1$, then $U_{q}(\mathscr{G}(A))$ has a PoincaréBirkhoff-Witt type basis.

Let $R$ be a commutative ring with 1 . Denote by $s l_{N+1}(R)$, the Lie algebra of $(N+1) \times(N+1)$ matrices over $R$ of trace 0 . It has the standard $R$-basis consisting of the elements

$$
e_{i, j}=E_{i, j}, f_{i, j}=E_{j, i}(1 \leq i<j \leq N+1), h_{i}=E_{i, i}-E_{i+1, i+1}(1 \leq i \leq N)
$$

( $E_{i, j}$ is the matrix having 1 in $(i, j)$ position and 0 elsewhere). By the PoincaréBirkhoff-Witt theorem [1], the elements

$$
f_{m_{1}, n_{1}} \cdots f_{m_{s}, n_{s}} h_{1}^{r_{1}} \cdots h_{N}^{r_{N}} e_{i_{1}, j_{1}} \cdots e_{i_{t}, j_{t}}
$$

$\left(r_{1}, \ldots, r_{N} \geq 0 ;\left(m_{1}, n_{1}\right) \leq \cdots \leq\left(m_{s}, n_{s}\right)\right.$ and $\left(i_{1}, j_{1}\right) \leq \cdots \leq\left(i_{s}, j_{s}\right)$ with respect to the lexicographic order $\leq$ ) form an $R$-basis of $U\left(s l_{N+1}(R)\right)$. Let $U_{q}\left(s l_{N+1}(R)\right)$ be the quantum group over $R$ associated with the Cartan matrix of type $A$. (See the beginning of Section 6 for the definition of $U_{q}\left(s l_{N+1}(R)\right)$. ) Let $R^{\times}$be the unit group of $R$. In this paper, for $q \in R^{\times}$such that $q^{8}-1 \in R^{\times}$, we construct an $R$ basis of $U_{q}\left(s l_{N+1}(R)\right)$ which can be considered as a natural $q$-analogue of $(*)$ (Theorem 1.1 and 6.1). Here the condition $q^{8}-1 \in R^{\times}$is essential. In fact,

Communicated by T. Kawai, April 17, 1989.

* Department of Mathematics, Faculty of Science, Osaka University, Toyonaka, Osaka 560, Japan. 
(i) When $R$ is a field and $q^{8}=1, U_{q}\left(s l_{N+1}(R)\right)$ seems to have no basis $q$-analogous to $(*)$. (Even in this case, we can give an explicit basis. See Proposition 6.2.)

(ii) If $q$ is an indeterminate and $R=\mathbb{C}\left[q^{\mp 1},\left(q^{4}-1\right)^{-1}\right]$, then the $R$-module $U_{q}\left(s l_{N+1}(R)\right)$ is not free. (See Proposition 6.3.)

To remedy this unpleasant situation, we are naturally led to introduce a new quantum group $\bar{U}_{q}\left(s l_{N+1}(R)\right)$ (Section 6$)$, which seems to be more natural than $U_{q}\left(s l_{N+1}(R)\right)$ in the following sense (See Theorem 6.1):

(o) $\quad \bar{U}_{q}\left(s l_{N+1}(R)\right)=U_{q}\left(s l_{N+1}(R)\right) \quad$ if $q^{8}-1 \in R^{\times}$,

(i) $\bar{U}_{q}\left(s l_{N+1}(R)\right)$ has an $R$-basis $q$-analogous to $(*)$.

We can define a filtration in $\bar{U}_{q}\left(s l_{N+1}(R)\right)$ such that the associated graded algebra is a non-commutative analogue of a polynomial ring (Section 5 (and 6)). As a corollary of this fact, we show that if $R$ is a Noetherian ring, then $\bar{U}_{q}\left(s l_{N+1}(R)\right)$ is a left (right) Noetherian ring, and that, if $R$ has no zero divisors $\neq 0$, then $\bar{U}_{q}\left(s l_{N+1}(R)\right)$ has no zero divisors $\neq 0$ (Theorem 1.2 and 6.1).

An important step in proving our main results is to show that a quantum group $U_{q}(\mathscr{G}(A))$ has a "triangular decomposition"; this is done in Section 2 for a general $A$. We also need " $\left(q\right.$-)commutator relations" in $U_{q}\left(s l_{N+1}(\mathbb{C})\right)($ Section 3$)$, which have been communicated to the author by Professor M. Jimbo. The author is very grateful to him.

In the last section, we also give an explicit Poincaré-Birkhoff-Witt basis of $U_{q}\left(\mathrm{SO}_{5}(\mathbb{C})\right)$.

\section{$\S 1$. Statement of the Main Results}

Let $A=\left(a_{i j}\right)_{1 \leq i, j \leq N}$ be a symmetrizable generalized Cartan matrix (see [8]); there exists a diagonal matrix $D=\operatorname{diag}\left(d_{1}, \ldots, d_{N}\right)$ such that $d_{i} \in \mathbb{Z} \backslash\{0\}$ and $D A$ $={ }^{t}(D A)$. Let $F$ be a field, and let $q \in F^{\times}$be such that $q^{4 d_{2}} \neq 0(1 \leq i \leq N)$. Let $U_{q}(\mathscr{G}(A))=U_{q}(\mathscr{G}(A), D)$ be the associative $F$-algebra with 1 with generators $e_{i}, f_{i}$, $k_{i}^{ \pm 1}(1 \leq i \leq N)$, and relations:

$$
\begin{aligned}
& k_{i} k_{i}^{-1}=k_{i}^{-1} k_{i}=1, k_{i} k_{j}=k_{j} k_{i} \\
& k_{i} e_{j} k_{i}^{-1}=q^{d_{\imath} a_{\imath \jmath}} e_{j}, k_{i} f_{j} k_{i}^{-1}=q^{-d_{\imath} a_{\imath \jmath}} f_{j} \\
& e_{i} f_{j}-f_{j} e_{i}=\delta_{i j} \frac{k_{i}^{2}-k_{i}^{-2}}{q^{2 d_{i}}-q^{-2 d_{i}}} \\
& \sum_{v=0}^{1-a_{t_{j}}}(-1)^{v}\left[\begin{array}{c}
1-a_{i j} \\
v
\end{array}\right]_{q^{2 d_{i}}} e_{i}^{1-a_{t \jmath}-v} e_{j} e_{i}^{v}=0 \quad(i \neq j) \\
& \sum_{v=0}^{1-a_{\imath j}}(-1)^{v}\left[\begin{array}{c}
1-a_{i j} \\
v
\end{array}\right]_{q^{2 d_{i}}} f_{i}^{1-a_{l j}-v} f_{j} f_{i}^{\nu}=0 \quad(i \neq j)
\end{aligned}
$$


where, for any two integers $m \geq n \geq 0$ and an arbitrary parameter $t$, $\left[\begin{array}{c}m \\ n\end{array}\right]_{t} \in \mathbb{Z}\left[t, t^{-1}\right]$ is defined by

$$
\left[\begin{array}{c}
m \\
n
\end{array}\right]_{t}= \begin{cases}\prod_{i=1}^{n} \frac{t^{m-i+1}-t^{-m+i-1}}{t^{i}-t^{-i}} & \text { if } m>n>0, \\
1 & \text { if } n=0 \text { or } m=n .\end{cases}
$$

When $A$ is the Cartan matrix of type $A_{N}$, we put $U_{q}\left(s l_{N+1}(F)\right)=U_{q}(\mathscr{G}(A)$, $\operatorname{diag}(1,1, \ldots, 1))$ for $q^{4} \neq 1$. For $1 \leq i<j \leq N+1$, we define inductively the elements $e_{i j}, f_{i j}$ of $U_{q}\left(s l_{N+1}(F)\right)$ by

$$
\begin{aligned}
& e_{i, i+1}=e_{i}, f_{i, i+1}=f_{i}, \\
& e_{i j}=q e_{i, j-1} e_{j-1, j}-q^{-1} e_{j-1, j} e_{i, j-1},(j-i>1)
\end{aligned}
$$

and

$$
f_{i j}=q f_{i, j-1} f_{j-1, j}-q^{-1} f_{j-1, j} f_{i, j-1},(j-i>1) .
$$

(The elements $e_{i j}$, $f_{i j}$ were introduced by Jimbo [7].)

Define the lexicographic order $<$ on $\mathbb{Z} \times \mathbb{Z}$ by

$$
(i, j)<(m, n) \text { if } i<m \text { or if } i=m, j<n .
$$

Now we can state our main theorem.

Theorem 1.1. Let $q \in F^{\times}$be such that $q^{8} \neq 1$. Then the elements

$$
f_{m_{1}, n_{1}} \cdots f_{m_{s}, n_{s}} k_{1}^{\ell} \cdots k_{N}^{\ell N} e_{i_{1}, j_{1}} \cdots e_{i_{t}, j_{t}}
$$

$\left(\left(m_{1}, n_{1}\right) \leq \cdots \leq\left(m_{s}, n_{s}\right), \quad\left(i_{1}, j_{1}\right) \leq \cdots \leq\left(i_{t}, j_{t}\right), \ell_{1}, \ldots, \ell_{N} \in \mathbb{Z}\right)$ form a basis of $U_{q}\left(s l_{N+1}(F)\right)$.

Theorem 1.2. If $q^{8} \neq 1$, then $U_{q}\left(s l_{N+1}(F)\right)$ is a left (right) Noetherian ring, and has no zero divisors $\neq 0$.

Remark. If $N \geq 3$ and $q$ is a primitive 8-th root of unity, the set of elements given in Theorem 1.1 does not span $U_{q}\left(s l_{N+1}(F)\right)$. Even in that case we can give an explicit basis of $U_{q}\left(s l_{N+1}(F)\right)$. See Proposition 6.2.

\section{$\S 2$. The Triangular Decomposition of $U_{q}(\mathscr{G}(A))$}

Let $A=\left(a_{i j}\right)_{1 \leq i, j \leq N}$ be a symmetrizable generalized Cartan matrix. Let $\tilde{U}_{q}\left(\mathscr{G}(A), \operatorname{diag}\left(d_{1}, \ldots, d_{N}\right)\right)$ be the associative $F$-algebra with 1 with generators $e_{i}$, $f_{i}, k_{i}^{ \pm 1}\left(1 \leq i \leq N\right.$ ), and relations (1.1), (1.2), (1.3). Let $\mathscr{X}_{+}$(resp. $\mathscr{X}_{-}$) be the free assoclative $F$-algebra with 1 with generators $\zeta_{1}, \ldots, \zeta_{N}$ (resp. $\xi_{1}, \ldots, \xi_{N}$ ). Let $F\left[v_{1}^{ \pm 1}, \ldots, v_{N}^{ \pm 1}\right]$ be the $F$-algebra of Laurent polynomials in indeterminates 
$v_{1}, \ldots, v_{N}$. Let $\mathfrak{M}=\mathscr{X}_{-} \otimes_{F} F\left[v_{1}^{ \pm 1}, \ldots, \quad v_{N}^{ \pm N}\right] \otimes_{F} \mathscr{X}_{+} . \quad$ Then the elements $\xi_{i_{1}} \ldots \xi_{i_{s}} v_{1}^{\ell} \ldots v_{N}^{\ell N} \zeta_{j_{1}} \cdots \zeta_{j_{t}}\left(\ell_{1}, \ldots, \ell_{N} \in \mathbb{Z}, 1 \leq i_{1}, \ldots, i_{s}, j_{1}, \ldots, j_{t} \leq N\right)$ form an $F-$ basis of $\mathfrak{M}$.

Lemma 2.1. $\mathfrak{M}$ has a left $\tilde{U}_{q}\left(A, \operatorname{diag}\left(d_{1}, \ldots, d_{N}\right)\right)$-module structure defined by

$$
\begin{aligned}
& k_{r} \cdot \xi_{i_{1}} \cdots \xi_{i_{s}} v_{1}^{\ell 1} \cdots v_{N}^{\ell N} \zeta_{j_{1}} \cdots \zeta_{j_{t}} \\
& =q^{-d_{r}\left(a_{r, i_{1}}+\cdots+a_{r} t_{s}\right)} \xi_{i_{1}} \cdots \xi_{i_{s}} v_{1}^{\ell 1} \cdots v_{r}^{\ell r+1} \cdots u_{N}^{\ell N} \zeta_{j_{1}} \cdots \zeta_{j_{t}} \\
& f_{r} \cdot \xi_{i_{1}} \cdots \xi_{i_{s}} v_{1}^{\ell 1} \cdots v_{N}^{\ell N} \zeta_{j_{1}} \cdots \zeta_{j_{t}} \\
& =\xi_{r} \xi_{i_{1}} \cdots \xi_{i_{s}} v_{1}^{\ell} \cdots v_{N}^{\ell N} \zeta_{j_{1}} \cdots \zeta_{j_{t}} \\
& e_{r} \cdot \xi_{i_{1}} \cdots \xi_{i_{s}} v_{1}^{\ell} \cdots v_{N}^{\ell N} \zeta_{j_{1}} \cdots \zeta_{j_{t}} \\
& =q^{-d_{r}\left(\ell_{1} a_{r, 1}+\cdots+\ell_{N} a_{r, N}\right)} \xi_{i_{1}} \cdots \zeta_{i_{s}} v_{1}^{\ell-1} \cdots v_{N}^{\ell N} \zeta_{r} \zeta_{j_{1}} \cdots \zeta_{j_{t}} \\
& +\frac{1}{q^{2 d_{r}}-q^{-2 d_{r}}} \sum_{i_{u}=r}\left\{q^{-2 d_{r} \alpha_{u}} \xi_{i_{1}} \cdots \xi_{i_{u}} \cdots \xi_{i_{s}} v_{1}^{\ell_{1}} \cdots v_{r}^{\ell_{r}+2} \cdots v_{N}^{\ell_{N}} \zeta_{j_{1}} \cdots \zeta_{j_{t}}\right. \\
& \left.-q^{2 d_{r} \alpha_{u}} \xi_{i_{1}} \cdots \xi_{i_{u}} \cdots \xi_{i_{s}} v_{1}^{\ell 1} \cdots v_{r}^{\ell_{r}-2} \cdots v_{N}^{\ell N} \zeta_{j_{1}} \cdots \zeta_{j_{t}}\right\}
\end{aligned}
$$

where $\alpha_{u}=a_{r, i_{u+1}}+a_{r, i_{u+2}}+\cdots+a_{r, i_{s}}$, and $\xi_{i_{u}}$ means that $\xi_{i_{u}}$ is omitted.

This can be verified by straightforward computations.

Lemma 2.2. The elements $f_{i_{1}} \cdots f_{i_{s}} k_{1}^{\ell^{1}} \cdots k_{N}^{\ell N} e_{j_{1}} \cdots e_{j_{t}} \quad\left(\ell_{1}, \ldots, \ell_{N} \in \mathbb{Z}\right.$, $\left.1 \leq i_{1}, \cdots, i_{s}, j_{1}, \cdots, j_{t} \leq N\right)$ form a basis of $\tilde{U}_{q}\left(A, \operatorname{diag}\left(d_{1}, \cdots, d_{N}\right)\right)$.

Proof. Let $1_{\mathfrak{m}} \in \mathfrak{M}$ (resp. $1_{\tilde{U}} \in \widetilde{U}_{q}(\mathscr{G}(A))$ be the unit element of $F\left[v_{1}^{ \pm 1}, \ldots, v_{N}^{ \pm 1}\right]$ (resp. $\left.\tilde{U}_{q}(\mathscr{G}(A))\right)$. By Lemma 2.1, we can define the left $\tilde{U}_{q}(\mathscr{G}(A))$-module homomorphisms $\quad \sigma: \quad \mathfrak{M} \rightarrow U_{q}(\mathscr{G}(A)) \quad$ and $\quad \tau: \quad \tilde{U}_{q}(\mathscr{G}(A)) \rightarrow \mathfrak{M} \quad$ by $\sigma\left(\xi_{i_{1}} \cdots \xi_{i_{s}} v_{1}^{\ell} \cdots v_{N}^{\ell N} \zeta_{j_{1}} \cdots \zeta_{j_{t}}\right)=f_{i_{1}} \cdots f_{i_{s}} k_{1}^{\ell} \cdots k_{N}^{\ell N} e_{j_{1}} \cdots e_{j_{t}}$ and $\tau(x)=x .1_{\mathfrak{M}}$. Then $\tau \circ \sigma$ is the identity map. Moreover, $\widetilde{U}_{q}(\mathscr{G}(A))=\widetilde{U}_{q}(\mathscr{G}(A)) .1_{\tilde{U}}=\tilde{U}_{q}(\mathscr{G}(A)) . \sigma\left(1_{\mathfrak{m}}\right)$ $=\sigma(\mathfrak{M})$. Hence $\sigma$ is bijective. Hence the lemma follows.

We prepare some notations which will be used hereafter.

$\cdot U_{q}\left(\mathcal{N}_{+}\right)\left(\right.$resp. $\left.\tilde{U}_{q}\left(\mathcal{N}_{+}\right)\right)$is the subalgebra of $U_{q}(\mathscr{G}(A))$ (resp. $\left.\tilde{U}_{q}(\mathscr{G}(A))\right)$ generated by the $e_{i}$ 's along with 1 .

$\cdot U_{q}\left(\mathscr{N}_{-}\right)\left(\right.$resp. $\left.\tilde{U}_{q}\left(\mathscr{N}_{-}\right)\right)$is the subalgebra of $U_{q}(\mathscr{G}(A))$ (resp. $\left.\tilde{U}_{q}(\mathscr{G}(A))\right)$ generated by the $f_{i}^{\prime}$ s along with 1 . $k_{i}^{ \pm 1} s$.

$\cdot H($ resp. $\widetilde{H})$ is the subalgebra of $U_{q}(\mathscr{G}(A))\left(\right.$ resp. $\left.\widetilde{U}_{q}(\mathscr{G}(A))\right)$ generated by the

$\cdot \phi_{i j}^{+}, \phi_{i j}^{-}(1 \leq i \neq j \leq N)$ are the elements of $\tilde{U}_{q}\left(\mathscr{G}(A), \operatorname{diag}\left(d_{1}, \ldots, d_{N}\right)\right)$ defined by 


$$
\begin{aligned}
& \phi_{i j}^{+}=\sum_{\nu=0}^{1-a_{\imath \jmath}}(-1)^{v}\left[\begin{array}{c}
1-a_{i j} \\
v
\end{array}\right]_{q 2 d_{i}} e_{i}^{1-a_{i j}-v} e_{j} e_{i}^{v}, \\
& \phi_{i j}^{-}=\sum_{\nu=0}^{1-a_{\imath j}}(-1)^{v}\left[\begin{array}{c}
1-a_{i j} \\
v
\end{array}\right]_{q 2 d_{l}} f_{i}^{1-a_{\imath \jmath}-v} f_{j} f_{i}^{v} .
\end{aligned}
$$

$\cdot I_{+}\left(\right.$resp. $\left.I_{-}\right)$is the two sided ideal of $\tilde{U}_{q}\left(\mathscr{N}_{+}\right)\left(\right.$resp. $\left.\tilde{U}_{q}\left(\mathscr{N}_{-}\right)\right)$generated by the $\phi_{i j}^{+}$'s (resp. the $\phi_{i j}^{-}$'s).

$\cdot K$ is the two sided ideal of $\widetilde{U}_{q}(\mathscr{G}(A))$ generated by the $\phi_{i j}^{+}$'s and $\phi_{i j}^{-}$'s.

Obviously, $U_{q}(\mathscr{G}(A), D) \simeq \tilde{U}_{q}(\mathscr{G}(A), D) / K$ as $F$-algebras. By Lemma 2.2, we have $\tilde{U}_{q}(\mathscr{G}(A)) \simeq \tilde{U}_{q}\left(\mathcal{N}_{-}\right) \otimes_{F} \tilde{H} \otimes_{F} \tilde{U}_{q}\left(\mathscr{N}_{+}\right)$as vector spaces, and $\tilde{U}_{q}\left(\mathcal{N}_{+}\right)$ $\simeq \mathscr{X}_{+} \simeq \mathscr{X}_{-} \simeq \tilde{U}_{q}\left(\mathscr{N}_{-}\right)$as $F$-algebras.

The following proposition gives a triangular decomposition of $U_{q}(\mathscr{G}(A))$.

Proposition 2.3. $\quad U_{q}(\mathscr{G}(A), D) \simeq U_{q}\left(\mathscr{N}_{-}\right) \bigotimes_{F} H \bigotimes_{F} U_{q}\left(\mathscr{N}_{+}\right)$as vector spaces. $U_{q}\left(\mathscr{N}_{ \pm}\right) \simeq \widetilde{U}_{q}\left(\mathcal{N}_{ \pm}\right) / I \pm$ as $F$-algebras. The elements $k_{1}^{\ell^{1}} \cdots k_{N}^{\ell N}\left(\ell_{1}, \ldots, \ell_{N} \in \mathbf{Z}\right)$ form a basis of $H$.

Proof. By Lemma 2.2, it suffices to prove:

$$
K=\left(\widetilde{U}_{q}\left(\mathscr{N}_{-}\right)\right) \tilde{H} I_{+}+I_{-} \tilde{H}\left(\tilde{U}_{q}\left(\mathscr{N}_{+}\right)\right) .
$$

This can be done by showing that $\left(\widetilde{U}_{q}\left(\mathscr{N}_{-}\right)\right) \tilde{H} I_{+}$and $I_{-} \tilde{H}\left(\tilde{U}_{q}\left(\mathscr{N}_{+}\right)\right)$are ideals of $\tilde{U}_{q}(\mathscr{G}(A), D)$. We only consider $I_{-} \tilde{H}\left(\tilde{U}_{q}\left(\mathcal{N}_{+}\right)\right)$, the argument for $\left(\tilde{U}_{q}\left(\mathscr{N}_{+}\right)\right) \tilde{H} I_{+}$ being analogous. Let $Y=I_{-} \widetilde{H}\left(\tilde{U}_{q}\left(\mathcal{N}_{+}\right)\right)$. It is clear that $k_{i}^{ \pm 1} Y \subset Y, Y k_{i}^{ \pm 1} \subset Y$ $f_{i} Y \subset Y, Y f_{i} \subset Y, Y e_{i} \subset Y$. The proof of $e_{i} Y \subset Y$ is similar to that of [9, Lemma 2.3] and as follows. Let $e_{i}^{ \pm}: \tilde{U}_{q}\left(\mathcal{N}_{-}\right) \rightarrow \tilde{U}_{q}\left(\mathcal{N}_{-}\right)$be the two $F$-linear maps defined by

$$
e_{i}^{ \pm}\left(f_{i_{1}} \cdots f_{i_{s}}\right)=\sum_{i_{u}=i} q^{ \pm 2 d_{2} \alpha_{u}} f_{i_{1}} \cdots \hat{f_{i_{u}}} \cdots f_{i_{s}},
$$

where $\alpha_{u}$ is an in (2.3), so that

$$
\begin{aligned}
& e_{i} \cdot f_{i_{1}} \cdots f_{i_{s}} k_{1}^{\ell} \cdots k_{N}^{\ell} N e_{j_{1}} \cdots e_{j_{t}} \\
& =q^{-d_{l}\left(\ell a_{1,1}+\cdots+\ell_{N} a_{i, N}\right)} f_{i_{1}} \cdots f_{i_{s}} k_{1}^{\ell 1} \cdots k_{N}^{\ell N} e_{i} e_{j_{1}} \cdots e_{j_{t}} \\
& +\frac{1}{q^{2 d_{t}}-q^{-2 d_{i}}} \sum_{i_{u}=i}\left\{e_{i}^{-}\left(f_{i_{1}} \cdots f_{i_{s}}\right) k_{1}^{\ell_{1}} \cdots k_{i}^{\ell^{2}+2} \cdots k_{N}^{\ell N} e_{j_{1}} \cdots e_{j_{t}}\right. \\
& \left.-e^{+}\left(f_{i_{1}} \cdots f_{i_{s}}\right) k_{1}^{\ell} \cdots k_{i}^{\ell}-2 \cdots k_{N}^{\ell N} e_{j_{1}} \cdots e_{j_{t}}\right\} \text {. }
\end{aligned}
$$

But we have

$$
\begin{aligned}
& e_{i}^{ \pm}\left(f_{i_{1}} \cdots f_{i_{p}} \phi_{\ell m}^{-} f_{i_{s}} \cdots f_{i_{s+\ell}}\right) \\
& \quad=\beta e_{i}^{ \pm}\left(f_{i_{1}} \cdots f_{i_{p}}\right) \phi_{\ell m}^{-} f_{i_{s}} \cdots f_{i_{s+\ell}}
\end{aligned}
$$




$$
\begin{aligned}
& +\beta^{\prime} f_{i_{1}} \cdots f_{i_{p}} e_{i}^{ \pm}\left(\phi_{\ell m}^{-}\right) f_{i_{s}} \cdots f_{i_{s+\ell}} \\
& +f_{i_{1}} \cdots f_{i_{p}} \phi_{\ell m}^{-} e_{i}^{ \pm}\left(f_{i_{s}} \cdots f_{i_{s+\ell}}\right)
\end{aligned}
$$

$\left(\beta, \beta^{\prime} \in F^{\times}, 1 \leq \ell \neq m \leq N\right)$. Hence it is enough to show that $e_{i}^{ \pm}\left(\phi_{\ell m}^{-}\right)=0$. If $i \neq \ell, m$, this is obvious. We consider the case $i=m$.

$$
\begin{aligned}
e_{l}^{ \pm}\left(\phi_{\ell i}^{-}\right) & \\
= & \left(\sum_{\nu=0}^{1-a_{\ell \imath}}(-1)^{v}\left[\begin{array}{c}
1-a_{\ell i} \\
v
\end{array}\right]_{q^{2 d_{\ell}}} q^{ \pm 2 d_{\ell} \cdot v a_{\ell \ell}}\right) f_{\ell}^{1-a_{\ell \ell}} \\
= & \left(\sum_{\nu=1}^{1-a_{\ell}}(-1)^{v}\left[\begin{array}{c}
1-a_{\ell i} \\
v-1
\end{array}\right]_{q^{2 d_{\ell}}} q^{ \pm 2 d_{\ell}(v-1)\left(a_{\ell \ell}-1\right)}\right. \\
& \left.+\sum_{\nu=0}^{-a_{\ell 2}}(-1)^{v}\left[\begin{array}{c}
1-a_{\ell i} \\
v
\end{array}\right]_{q^{2 d_{\ell}}} q^{ \pm 2 d_{\ell} \cdot v\left(a_{\ell \ell}-1\right)}\right) f_{\ell}^{1-a_{\ell \ell}} \\
= & 0 .
\end{aligned}
$$

In the above computation, we used the formulas:

$$
\left[\begin{array}{c}
m \\
n
\end{array}\right]_{t}=t^{ \pm(m-n)}\left[\begin{array}{c}
m-1 \\
n-1
\end{array}\right]_{t}+t^{ \pm n}\left[\begin{array}{c}
m-1 \\
n
\end{array}\right]_{t}(m>n>1) .
$$

The remaining case $i=\ell$ can be verified by a direct computation.

Remark. Proposition 2.3 is an extention of [11, Prop. 2].

Corollary 2.4. Let $A=\left(a_{i j}\right)_{1 \leq i, j \leq N}$ be the symmetrizable Cartan matrix. For $1 \leq M \leq N$, let $A^{\prime}=\left(a_{i j}\right)_{1 \leq i, j \leq M}$ be the submatrix of $A$. Then the subalgebra of $U_{q}\left(\mathscr{G}(A), \operatorname{diag}\left(d_{1}, \ldots, d_{N}\right)\right)$ generated by $\left\{e_{i}, f_{i}, k_{i}^{ \pm 1} \mid 1 \leq i \leq M\right\}$ is isomorphic to $U_{q}\left(\mathscr{G}\left(A^{\prime}\right), \operatorname{diag}\left(d_{1}, \ldots, d_{M}\right)\right)$ (as Hopf algebras).

Proof. Let $U_{q}\left(\mathcal{N}_{-}^{\prime}\right) \bigotimes_{F} H \otimes_{F} U_{q}\left(\mathscr{N}_{+}^{\prime}\right)$ be the triangular decomposition of $U_{q}\left(\mathscr{G}\left(A^{\prime}\right)\right.$, $\left.\operatorname{diag}\left(d_{1}, \ldots, d_{M}\right)\right)$. Define the two homomorphisms $i_{ \pm}: U_{q}\left(\mathscr{N}_{ \pm}^{\prime}\right)$ $\rightarrow U_{q}\left(\mathcal{N}_{ \pm}\right)$by $i_{+}\left(e_{i}\right)=e_{i}$ and $i_{-}\left(f_{i}\right)=f_{i}(1 \leq i \leq M)$. By Proposition 2.3, it suffices to show that $i_{ \pm}$are injective. We consider $i_{+}$. By Proposition 2.3, we can define the homomorphism $p_{+}: U_{q}\left(\mathscr{N}_{+}\right) \rightarrow U_{q}\left(\mathscr{N}_{+}^{\prime}\right)$ by $p_{+}\left(e_{i}\right)=e_{i}(1 \leq i \leq M)$ and $p_{+}\left(e_{i}\right)=0(M<i \leq N)$. It is clear that $p_{+} \circ i_{+}$is the identity map. Hence it is injective.

\section{§3. Some (q-) commutator Relations in $U_{q}\left(s l_{N+1}(F)\right)$}

From now on, until the end of $\S 5$, we are concerned with the quantum group $U_{q}\left(s l_{N+1}(F)\right), q^{8} \neq 1$. For a positive integer $N$, put $\Lambda_{N}=\{(i, j) \in \mathbb{Z} \times \mathbb{Z} \mid 1 \leq i$ $<j \leq N+1\}$. For $(i, j),(m, n) \in \Lambda_{N}$ such that $(i, j)<(m, n)(\operatorname{see}(1.7))$, there are following six cases: 


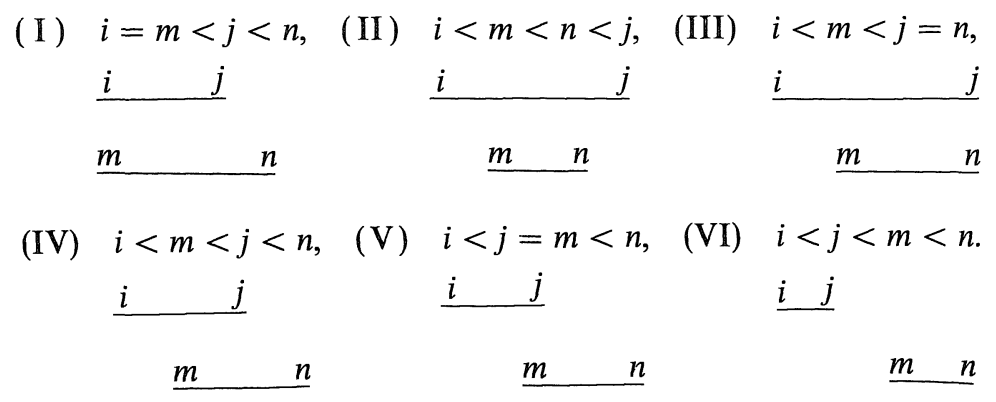

Set

$$
\begin{aligned}
& C_{(\mathrm{I})}=\left\{((i, j),(m, n)) \in \Lambda_{N} \times \Lambda_{N} \mid i=m<j<n\right\}, \\
& C_{(\mathrm{II})}=\left\{((i, j),(m, n)) \in \Lambda_{N} \times \Lambda_{N} \mid i<m<n<j\right\}, \\
& C_{(\mathrm{III})}=\left\{((i, j),(m, n)) \in \Lambda_{N} \times \Lambda_{N} \mid i<m<j=n\right\}, \\
& C_{(\mathrm{IV})}=\left\{((i, j),(m, n)) \in \Lambda_{N} \times \Lambda_{N} \mid i<m<j<n\right\}, \\
& C_{(\mathrm{V})}=\left\{((i, j),(m, n)) \in \Lambda_{N} \times \Lambda_{N} \mid i<j=m<n\right\}, \\
& C_{(\mathrm{VI})}=\left\{((i, j),(m, n)) \in \Lambda_{N} \times \Lambda_{N} \mid i<j<m<n\right\} .
\end{aligned}
$$

When $q^{8} \neq 1$, we get the following formulas. We denote by $e_{i j}$ and $f_{m n}$ the elements of $U_{q}\left(s l_{N+1}(F)\right)$ defined in $(1,6)$, and by $[x, y]$ the usual commutator $x y-y x$.

(1)

$$
\begin{array}{ll}
q^{-2} e_{i j} e_{m n}-e_{m n} e_{i j}=0 & \text { if }((i, j),(m, n)) \in C_{(\mathrm{I})} \cup C_{(\mathrm{III})} \\
{\left[e_{i j}, e_{m n}\right]=0} & \text { if }((i, j),(m, n)) \in C_{(\mathrm{II})} \cup C_{(\mathrm{VI})} \\
{\left[e_{i j}, e_{m n}\right]=\left(q^{2}-q^{-2}\right) e_{i n} e_{m j}} & \text { if }((i, j),(m, n)) \in C_{(\mathrm{IV})} \\
q^{2} e_{i j} e_{m n}-e_{m n} e_{i j}=q e_{i n} & \text { if }((i, j),(m, n)) \in C_{(\mathrm{V})}
\end{array}
$$

(2) The $f_{i j}$ 's also satisfy relations similar to (1).

(3)

$$
\begin{aligned}
& {\left[e_{i j}, f_{m n}\right]=(-1)^{j-i+1} q f_{j n} k_{i}^{2} k_{i+1}^{2} \cdots k_{j-1}^{2} \quad \text { if }((i, j),(m, n)) \in C_{(\mathrm{I})} .} \\
& {\left[e_{i j}, f_{m n}\right]=(-1)^{n-m+1} q k_{m}^{2} k_{m+1}^{2} \cdots k_{n-1}^{2} e_{i m} \quad \text { if }((i, j)),(m, n) \in C_{(\mathrm{III}) \cdot}} \\
& {\left[e_{i j}, f_{m n}\right]=(-1)^{j-m+1}\left(q^{4}-1\right) f_{j n} k_{m}^{2} k_{m+1}^{2} \cdots k_{j-1}^{2} e_{i m}} \\
& {\left[e_{i j}, f_{m n}\right]=0 \quad \text { if }((i, j),(m, n)) \in C_{(\mathrm{IV})}} \\
& {\left[e_{m n}, f_{i j}\right]=(-1)^{j-i} q^{-1} k_{i}^{-2} k_{i+1}^{-2} \cdots k_{j-1}^{-2} e_{j n} \text { if }((i, j),(m, n)) \in C_{(\mathrm{I})} .} \\
& {\left[e_{m n}, f_{i j}\right]=(-1)^{n-m} q^{-1} f_{j m} k_{m}^{-2} k_{m+1}^{-2} \cdots k_{n-1}^{-2} \text { if }((i, j),(m, n)) \in C_{(\mathrm{III}) \cdot}} \\
& {\left[e_{m n}, f_{i j}\right]=(-1)^{j-m}\left(1-q^{-4}\right) f_{i m} k_{m}^{-2} k_{m+1}^{-2} \cdots k_{j-1}^{-2} e_{j n} \text { if }((i, j),(m, n)) \in C_{(\mathrm{IV}) \cdot}} \\
& {\left[e_{m n}, f_{i j}\right]=0 \text { if }((i, j),(m, n)) \in C_{(\mathrm{II})} \cup C_{(\mathrm{V})} \cup C_{(\mathrm{VI})} .}
\end{aligned}
$$$$
\left[e_{i j}, f_{i j}\right]=\frac{(-1)^{j-i+1}}{q^{2}-q^{-2}}\left(k_{i}^{2} k_{i+1}^{2} \cdots k_{j-1}^{2}-k_{i}^{-2} k_{i+1}^{-2} \cdots k_{j-1}^{-2}\right) \text {. }
$$ 
(4)

$$
\begin{aligned}
& k_{r} e_{i j} k_{r}^{-1}=q^{\left(a_{i, r}+a_{l+1, r}+\ldots+a_{j-1, r}\right)} e_{i j} . \\
& k_{r} f_{i j} k_{r}^{-1}=q^{-\left(a_{i, r}+a_{l+1, r}+\ldots+a_{j-1, r}\right)} f_{i j} .
\end{aligned}
$$

Among these, we just prove:

$$
\begin{array}{ll}
q^{2} e_{i j} e_{m n}-e_{m n} e_{i j}=q e_{i n} & \text { if }((i, j),(m, n)) \in C_{(\mathrm{V})}, \\
{\left[e_{i j}, e_{m n}\right]=0} & \text { if }((i, j),(m, n)) \in C_{(\mathrm{II})} ;
\end{array}
$$

to verify other formulas is left to the reader. To prove the formula (3.1), we use induction on $n-m$ :

$$
\begin{aligned}
q^{2} & e_{i, j} e_{m, n}-e_{m, n} e_{i, j} \\
= & q^{2} e_{i, m}\left(q e_{m, n-1} e_{n-1, n}-q^{-1} e_{n-1, n} e_{m, n-1}\right) \\
& -\left(q e_{m, n-1} e_{n-1, n}-q^{-1} e_{n-1, n} e_{m, n-1}\right) e_{i, m} \\
= & q^{2} e_{i, n-1} e_{n-1, n}-e_{n-1, n} e_{i, n-1} \\
= & q e_{i, n} .
\end{aligned}
$$

We get the formula (3.2) by (3.1) and the following formula:

$$
\begin{aligned}
{\left[e_{i, i+3},\right.} & \left.e_{i+1, i+2}\right] \\
= & \left(q^{2} e_{i} e_{i+1} e_{i+2}-e_{i+1} e_{i} e_{i+2}-e_{i+2} e_{i} e_{i+1}+q^{-2} e_{i+2} e_{i+1} e_{i}\right) e_{i+1} \\
& -e_{i+1}\left(q^{2} e_{i} e_{i+1} e_{i+2}-e_{i+1} e_{i} e_{i+2}-e_{i+2} e_{i} e_{i+1}+q^{-2} e_{i+2} e_{i+1} e_{i}\right) \\
= & q^{2} e_{i}\left(\left(q^{2}+q^{-2}\right)^{-1}\left(e_{i+1}^{2} e_{i+2}+e_{i+2} e_{i+1}^{2}\right)\right)-e_{i+1} e_{i} e_{i+2} e_{i+1} \\
& \quad-e_{i+2} e_{i} e_{i+1}^{2}+q^{-2} e_{i+2}\left(\left(q^{2}+q^{-2}\right)^{-1}\left(e_{i+1}^{2} e_{i}+e_{i} e_{i+1}^{2}\right)\right) \\
& -q^{2}\left(\left(q^{2}+q^{-2}\right)^{-1}\left(e_{i+1}^{2} e_{i}+e_{i} e_{i+1}^{2}\right)\right) e_{i+2}+e_{i+1}^{2} e_{i} e_{i+2} \\
& +e_{i+1} e_{i+2} e_{i} e_{i+1}-q^{-2}\left(\left(q^{2}+q^{-2}\right)^{-1}\left(e_{i+1}^{2} e_{i+2}+e_{i+2} e_{i+1}^{2}\right)\right) e_{i} \\
= & 0 .
\end{aligned}
$$

We note that the condition $q^{8} \neq 1$ is used in proving (3.2).

\section{$\S 4$. Proof of Theorem 1.1}

Let $\Lambda_{N}$ be as in $\S 3$. Let $\mathcal{N}$ be an $F$-vector space spanned by a basis $\left\{\mathscr{X}_{i j} \mid(i, j) \in \Lambda_{N}\right\}$. Let $\mathscr{X}(\mathcal{N})$ be the free associative $F$-algebra with 1 with generators $x_{i j}\left((i, j) \in \Lambda_{N}\right)$. For $(i, j),(m, n) \in \Lambda_{N}$ such that $(i, j)<(m, n)$, we define $\varepsilon_{i j m n} \in F^{\times}$and $y_{i j m n} \in \mathscr{X}(\mathscr{N})$ by 
$\varepsilon_{i j m n}= \begin{cases}1 & \text { if }((i, j),(m, n)) \in C_{(\mathrm{II})} \cup C_{(\mathrm{IV})} \cup C_{(\mathrm{VI})} \\ q^{-2} & \text { if }((i, j),(m, n)) \in C_{(\mathrm{I})} \cup C_{(\mathrm{III})} \\ q^{2} & \text { if }((i, j),(m, n)) \in C_{(\mathrm{V})}\end{cases}$

$y_{i j m n}= \begin{cases}q x_{i n} & \text { if }((i, j),(m, n)) \in C_{(\mathrm{V})} \\ \left(q^{2}-q^{-2}\right) x_{i n} x_{m j} & \text { if }((i, j),(m, n)) \in C_{(\mathrm{IV})} \\ 0 & \text { if }((i, j),(m, n)) \in C_{(\mathrm{I})} \cup C_{(\mathrm{II})} \cup C_{(\mathrm{III})} \cup C_{(\mathrm{VI})},\end{cases}$

where $C_{(\mathrm{I})}, \ldots, C_{(\mathrm{VI})}$ are as in $\S 3$.

Let I (resp. $\mathbb{J}$ ) be the two sided ideal of $\mathscr{X}(\mathscr{N})$ generated by the elements $\varepsilon_{i j m n} x_{i j} x_{m n}-x_{m n} x_{i j}-y_{i j m n} \quad\left(\right.$ resp. $\left.\varepsilon_{i j m n} x_{i j} x_{m n}-x_{m n} x_{i j}\right)$ for $(i, j)<(m, n)$. Put $U_{q}(\mathscr{N})=\mathscr{X}(\mathscr{N}) / \mathbf{I}$ and $\widetilde{\Xi}_{q}(\mathscr{N})=\mathscr{X}(\mathcal{N}) / \mathbf{J}$. Let $\tilde{x}_{i j}=x_{i j}+\mathbb{I} \in U_{q}(\mathscr{N}), \tilde{y}_{i j m n}=y_{i j m n}$ $+\mathbf{I} \in U_{q}(\mathscr{N})$ and $z_{i j}=x_{i j}+\mathbf{J} \in \Xi_{q}(\mathscr{N})$.

Lemma 4.1. If $q^{8} \neq 1$, there exist isomorphisms $\varphi_{ \pm}: U_{q}(\mathcal{N}) \rightarrow U_{q}\left(\mathscr{N}_{ \pm}\right)$such that $\varphi_{+}\left(\tilde{x}_{i j}\right)=e_{i j}, \varphi_{-}\left(\tilde{x}_{i j}\right)=f_{i j}\left((i, j) \in \Lambda_{N}\right)$.

Proof. We only consider $\varphi_{+}$. By the formulas in $\S 3, \varphi_{+}$is welldefined. By the definition of $U_{q}(\mathscr{N})$, we have

$$
\tilde{x}_{i, i+1} \tilde{x}_{j, j+1}-\tilde{x}_{j, j+1} \tilde{x}_{i, i+1}=0 \quad \text { for }|i-j| \geq 2,
$$

and

$$
\begin{aligned}
& \left(\tilde{x}_{i, i+1}\right)^{2} \tilde{x}_{i+1, i+2}-\left(q^{2}+q^{-2}\right) \tilde{x}_{i, i+1} \tilde{x}_{i+1, i+2} \tilde{x}_{i, i+1}+\tilde{x}_{i+1, i+2}\left(\tilde{x}_{i, i+1}\right)^{2} \\
& =q^{-1} \tilde{x}_{i, i+1} \tilde{x}_{i, i+2}-q \tilde{x}_{i, i+2} \tilde{x}_{i, i+1}=0 .
\end{aligned}
$$

Similarly,

$$
\begin{aligned}
& \left(\tilde{x}_{i+1, i+2}\right)^{2} \tilde{x}_{i, i+1}-\left(q^{2}+q^{-2}\right) \tilde{x}_{i+1, i+2} \tilde{x}_{i, i+1} \tilde{x}_{i+1, i+2} \\
& \quad+\tilde{x}_{i, i+1}\left(\tilde{x}_{i+1, i+2}\right)^{2} \\
& =0 .
\end{aligned}
$$

Hence we can define the homomorphism $\psi_{+}: U_{q}\left(\mathscr{N}_{+}\right) \rightarrow U_{q}(\mathscr{N})$ by $\psi_{+}\left(e_{i}\right)$ $=\tilde{x}_{i, i+1}$. It is obvious that $\varphi_{+}{ }^{\circ} \psi_{+}$and $\psi_{+}{ }^{\circ} \varphi_{+}$are the identity maps.

Let $P_{N}$ be the set of finite sequences of elements of $\Lambda_{N}$. We consider the "empty sequence" $(\phi)$ is also an element of $P_{N}$. For $\Sigma=\left(\left(i_{1}, j_{1}\right), \ldots,\left(i_{t}, j_{t}\right)\right) \in P_{N}$, we put $x_{\Sigma}=x_{i_{1}, j_{1}} \cdots x_{i_{t}, j_{t}}$; we understand that $x_{(\phi)} 1$. Put $\tilde{x}_{\Sigma}=x_{\Sigma}+I \in U_{q}(\mathcal{N}), z_{\Sigma}$ $=x_{\Sigma}+\mathbf{J} \in \mathfrak{S}_{q}(\mathscr{N})$. Define the function $\eta: P_{N} \rightarrow \mathbf{Z}$ by $\eta(\Sigma)=i_{1}\left(j_{1}-i_{1}\right)+\cdots$ $+i_{t}\left(j_{t}-i_{t}\right)$ and $\eta((\phi))=0$. For a nonnegative integer $m$, let $U_{m}$ (resp. $S_{m}$ ) be the subspace of $U_{q}(\mathcal{N})\left(\operatorname{resp} . \subseteq_{q}(\mathcal{N})\right)$ spanned by the elements $\tilde{x}_{\Sigma}$ (resp. $z_{\Sigma}$ ) such that $\eta(\Sigma) \leq m$, along with 1 . Call $\Sigma$ increasing if $\left(i_{1}, j_{1}\right) \leq \cdots \leq\left(i_{t}, j_{t}\right) . \quad(\phi)$ is also considered to be increasing. 
Lemma 4.2. $U_{q}(\mathcal{N})$ is spanned by $\left\{\tilde{x}_{\Sigma} \mid \Sigma\right.$ increasing $\}$ as a vector space.

Proof. Assume that any element of $U_{m-1}$ is an $F$-linear combination of the $\tilde{x}_{\Sigma}{ }^{s} s$ such that $\eta(\Sigma) \leq m-1$ and that $\Sigma$ is increasing. It suffices to show that, for $\Sigma=\left(\left(i_{1}, j_{1}\right), \ldots,\left(i_{t}, j_{t}\right)\right)$ satisfying $\eta(\Sigma)=m$, we have

$$
\begin{aligned}
& x_{i_{1}, j_{1}} \cdots x_{i_{u}, j_{u}} x_{i_{u+1}, j_{u+1}} \cdots x_{i_{t}, j_{t}} \\
& \quad \equiv \varepsilon_{i_{u+1}, j_{u+1}, i_{u}, j_{u}} x_{i_{1}, j_{1}} \cdots x_{i_{u+1}, j_{u+1}} x_{i_{u}, j_{u}} \cdots x_{i_{t}, j_{t}} \\
& \left(\bmod U_{m-1}\right) .
\end{aligned}
$$

for an integer $u$ such that $\left(i_{u}, j_{u}\right)>\left(i_{u+1}, j_{u+1}\right)$. Indeed, if $\left(\left(i_{u+1}, j_{u+1}\right),\left(i_{u}\right.\right.$, $\left.\left.j_{u}\right)\right) \in C_{(\text {I })} \cup C_{(\text {II })} \cup C_{(\mathrm{III})} \cup C_{(\mathrm{VI})}$, this follows from (4.2). If it belongs to $C_{(\mathrm{V})}\left(\right.$ resp. $\left.C_{(\mathrm{IV})}\right)$, then it is obtained from (4.2) and the formula:

$$
\begin{aligned}
& \eta\left(\left(\left(i_{u+1}, j_{u+1}\right),\left(i_{u}, j_{u}\right)\right)\right)-\eta\left(\left(\left(i_{u+1}, j_{u}\right)\right)\right) \\
& =\left(i_{u}-i_{u+1}\right)\left(j_{u}-j_{u+1}\right)>0 \\
& \text { (resp. } \eta\left(\left(\left(i_{u+1}, j_{u+1}\right),\left(i_{u}, j_{u}\right)\right)\right)-\eta\left(\left(\left(i_{u+1}, j_{u}\right),\left(i_{u}, j_{u+1}\right)\right)\right) \\
& \left.=\left(i_{u}-i_{u+1}\right)\left(j_{u}-j_{u+1}\right)>0\right) .
\end{aligned}
$$

Now we show that $\left\{\tilde{x}_{\Sigma} \mid \Sigma\right.$ increasing $\}$ is, in fact, a basis of $U_{q}(\mathscr{N})$.

Lemma 4.3. The set $\left\{z_{\Sigma} \mid \sum\right.$ increasing $\}$ is a basis of $\mathfrak{S}_{q}(\mathcal{N})$.

The proof is similar to that of Lemma 2.2; instead of Lemma 2.1, we need the following Lemma 4.4. We omit the details.

Lemma 4.4. For $1 \leq i<j \leq n$, let $c_{i j} \in F$. We denote by $\mathscr{T}_{n}$ the associative $F$-algebra with generators $t_{1}, \ldots, t_{n}$ and relations $c_{i j} t_{i} t_{j}-t_{j} t_{i}$. Let $F\left[v_{1}, \ldots, v_{n}\right]$ be the $F$-algebra of polynomials in indeterminates $v_{1}, \ldots, v_{n}$. Then $F\left[v_{1}, \ldots, v_{n}\right]$ has a left $\mathscr{T}_{n}$-module structure defined by

$$
t_{i} \cdot v_{1}^{r_{1}} \cdots v_{n}^{r_{n}}=c_{1, i}^{r_{1}} c_{2, i}^{r_{2}} \cdots c_{i-1, i}^{r_{1}} i_{1}^{r_{1}} \cdots v_{i}^{r_{1}+1} \cdots v_{n}^{r_{n}} .
$$

For $\lambda, \mu \in \Lambda_{N}$ and $\Sigma \in P_{N}$, write $\lambda \leq \Sigma$ if $\lambda \leq \mu$ for all $\mu \in \Sigma$.

Lemma 4.5. There exists an F-bilinear map $f: \mathcal{N} \times \mathfrak{S}_{q}(\mathcal{N}) \rightarrow \mathfrak{S}_{q}(\mathcal{N})$ satisfying:

(A) $f\left(x_{\lambda}, z_{\Sigma}\right)=z_{\lambda} z_{\Sigma}$ for $\lambda \leq \Sigma$.

(B) $f\left(x_{\lambda}, z_{\Sigma}\right) \equiv z_{\lambda} z_{\Sigma}\left(\bmod S_{\eta(\Sigma)+\eta(\lambda)-1}\right)$.

(C) For all $(i, j)<(m, n)$,

$$
\varepsilon_{i j m n} f\left(x_{i j}, f\left(x_{m n}, z_{T}\right)\right)-f\left(x_{m n}, f\left(x_{i j}, z_{T}\right)\right)
$$




$$
=\left\{\begin{array}{cl}
q f\left(x_{i n}, z_{T}\right) & \text { if }((i, j),(m, n)) \in C_{(\mathrm{V})} \\
\left(q^{2}-q^{-2}\right) f\left(x_{i n}, f\left(x_{m j}, z_{T}\right)\right) & \text { if }((i, j),(m, n)) \in C_{(\mathrm{IV})} \\
0 & \text { if }((i, j),(m, n)) \in C_{(\mathrm{I})} \cup C_{(\mathrm{II})} \cup C_{(\mathrm{III})} \cup C_{(\mathrm{VI})} .
\end{array}\right.
$$

In order to prove this, we need:

Lemma 4.6. Let $r$ be a positive integer. Assume an $F$-bilinear map $f^{\prime}: \mathscr{N}$ $\times \mathfrak{S}_{q}(\mathcal{N}) \rightarrow \mathfrak{S}_{q}(\mathcal{N})$ satisfies the following:

(B) $f^{\prime}\left(x_{\lambda}, z_{\Sigma}\right) \equiv z_{\lambda} z_{\Sigma} \quad\left(\bmod S_{\eta(\Sigma)+\eta(\lambda)-1}\right)$.

$\left(\mathrm{C}^{\prime}\right)$ For all $(i, j),(m, n) \in \Lambda_{N}, T \in P_{N}$ such that $(i, j)<(m, n)$ and $\eta(i, j)+\eta$ $(m, n)+\eta(T)<r$,

$$
\begin{aligned}
\varepsilon_{i j m n} f^{\prime}\left(x_{i j}, f^{\prime}\left(x_{m n}, z_{T}\right)\right)-f^{\prime}\left(x_{m n}, f^{\prime}\left(x_{i j}, z_{T}\right)\right) \\
\quad= \begin{cases}q f^{\prime}\left(x_{i n}, z_{T}\right) & \text { if }((i, j),(m, n)) \in C_{(\mathrm{V})} \\
\left(q^{2}-q^{-2}\right) f^{\prime}\left(x_{i n}, f^{\prime}\left(x_{m j}, z_{T}\right)\right) & \text { if }((i, j),(m, n)) \in C_{(\mathrm{IV})} \\
0 & \text { if }((i, j),(m, n)) \in C_{(\mathrm{I})} \cup C_{(\mathrm{II})} \cup C_{(\mathrm{III})} \cup C_{(\mathrm{VI})} .\end{cases}
\end{aligned}
$$

Then, for $(h, \ell)<(i, j)<(m, n)$ and $\Psi \in P_{N}$ such that $\eta(h, \ell)+\eta(i, j)+\eta(m, n)$ $+\eta(\Psi) \leq r$, it follows:

$$
\begin{aligned}
\varepsilon_{h \ell i j} & \varepsilon_{h \ell m n} x_{h \ell} y_{i j m n} z_{\Psi}-y_{i j m n} x_{h \ell} z_{\Psi} \\
& -\varepsilon_{i j m n} x_{i j} y_{h \ell m n} z_{\Psi}+\varepsilon_{h \ell i j} y_{h \ell m n} x_{i j} z_{\Psi} \\
& +x_{m n} y_{h \ell i j} z_{\Psi}-\varepsilon_{i j m n} \varepsilon_{h \ell m n} y_{h \ell i j} x_{m n} z_{\Psi} \\
= & 0 .
\end{aligned}
$$

(Here we abbreviate $f^{\prime}\left(x_{i_{1}}, j_{1}, f^{\prime}\left(x_{i_{2}, j_{2}}, \ldots, f^{\prime}\left(x_{i_{t}, j_{t}}, z_{\Psi}\right)\right.\right.$ )) to $x_{i_{1}, j_{1}} x_{i_{2}, j_{2}} \cdots x_{i_{t}, j_{t}} z_{\Psi}$.)

Sketch of the proof. In $\S 3$, we have seen that there are 6 cases for $((i, j),(m$, $n)) \in \Lambda_{N} \times \Lambda_{N}$ such that $(i, j)<(m, n)$. Similarly we can see that there are 62 cases for $((h, \ell),(i, j),(m, n)) \in \Lambda_{N} \times \Lambda_{N} \times \Lambda_{N}$ such that $(h, \ell)<(i, j)<(m, n)$ (See Figure 1). 
Figure 1 (we omit the letters $m$ and $n$ ).

( I )

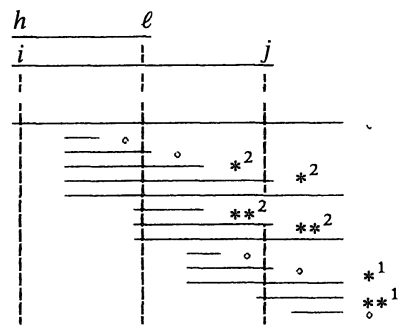

(III)

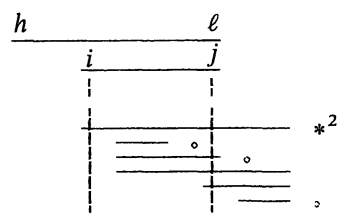

(V) $\underline{h \quad \ell}$

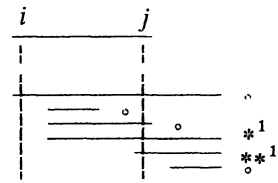

( II )

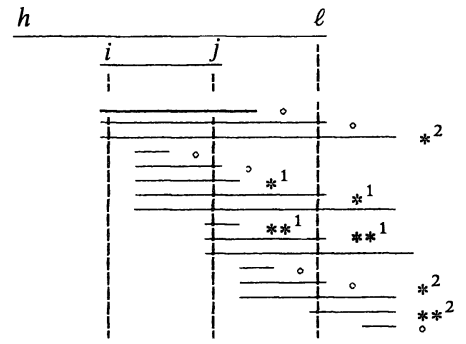

(IV)

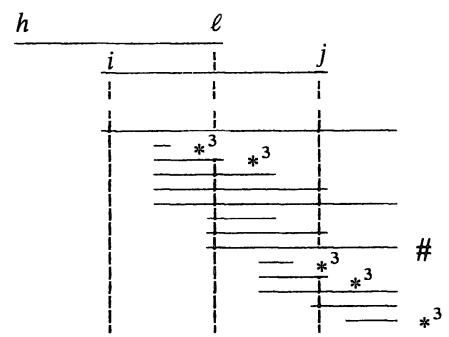

(VI)

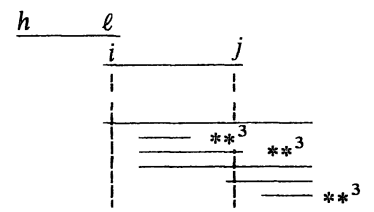

In the 20 cases labelled $\circ$, since $y_{i j m n}=y_{h \ell m n}=y_{h \ell i j}=0$, the formula (4.5) is obvious. In the 24 cases labelled $*^{r}$ or $* *^{r}(r=1,2,3)$, we have

$$
y_{i j m n}=\delta_{1 r}, y_{h \ell m n}=\delta_{2 r}, y_{h \ell i j}=\delta_{3 r} \text {. }
$$

In the cases of $*^{r}$,

the left-hand side of (4.5)

$$
\begin{aligned}
= & \varepsilon_{h \ell i j} \varepsilon_{h \ell m n} x_{h \ell} \delta_{1 r}\left(q^{2}-q^{-2}\right) x_{i n} x_{m j} z_{\Psi}-\delta_{1 r}\left(q^{2}-q^{-2}\right) x_{i n} x_{m j} x_{h \ell} z_{\Psi} \\
& -\varepsilon_{i j m n} x_{i j} \delta_{2 r}\left(q^{2}-q^{-2}\right) x_{h n} x_{m \ell} z_{\Psi}+\varepsilon_{h \ell i j} \delta_{2 r}\left(q^{2}-q^{-2}\right) x_{h n} x_{m \ell} x_{i j} z_{\Psi} \\
& +x_{m n} \delta_{3 r}\left(q^{2}-q^{-2}\right) x_{h j} x_{i \ell} z_{\Psi}-\varepsilon_{i j m n} \varepsilon_{h \ell m n} \delta_{3 r}\left(q^{2}-q^{-2}\right) x_{h j} x_{i \ell} x_{m n} z_{\Psi} \\
= & \left(q^{2}-q^{-2}\right)\left(\delta_{1 r}\left(\varepsilon_{h \ell i j} \varepsilon_{h \ell m n}-\varepsilon_{h \ell m j} \varepsilon_{h \ell i n}\right) x_{h \ell} x_{i n} x_{m j} z_{\Psi}\right. \\
& -\delta_{2 r}\left(\varepsilon_{i j m n} \varepsilon_{h n i j}-\varepsilon_{h \ell i j} \varepsilon_{i j m \ell}\right) x_{h n} x_{i j} x_{m \ell} z_{\Psi} \\
& \left.+\delta_{3 r}\left(\varepsilon_{h j m n} \varepsilon_{i \ell m n}-\varepsilon_{i j m n} \varepsilon_{h \ell m n}\right) x_{h j} x_{m n} x_{i \ell} z_{\Psi}\right) \\
= & 0 .
\end{aligned}
$$


The cases of $* *^{r}$, can be treated similarly.

In each of the remaining 18 cases, we can get (4.5) after easy computation. For example, in the case of $h<i<\ell=m<j<n$ (labelled \#),

the left-hand side of (4.5)

$$
\begin{aligned}
= & q^{2}\left(q^{2}-q^{-2}\right) x_{h \ell} x_{i n} x_{m j} z_{\Psi}-\left(q^{2}-q^{-2}\right) x_{i n} x_{m j} x_{h \ell} z_{\Psi} \\
& -q x_{i j} x_{h n} z_{\Psi}+q x_{h n} x_{i j} z_{\Psi} \\
& +\left(q^{2}-q^{-2}\right) x_{m n} x_{h j} x_{i \ell} z_{\Psi}-q^{2}\left(q^{2}-q^{-2}\right) x_{h j} x_{i \ell} x_{m n} z_{\Psi} \\
= & q^{2}\left(q^{2}-q^{-2}\right) x_{h \ell} x_{i n} x_{m j} z_{\Psi}-q^{2}\left(q^{2}-q^{-2}\right) x_{i n} x_{h \ell} x_{m j} z_{\Psi}+ \\
& q\left(q^{2}-q^{-2}\right) x_{i n} x_{h j} z_{\Psi} \\
& +\left(q^{2}-q^{-2}\right) x_{h j} x_{m n} x_{i \ell} z_{\Psi}-\left(q^{2}-q^{-2}\right)^{2} x_{h n} x_{m j} x_{i \ell} z_{\Psi}- \\
& q^{2}\left(q^{2}-q^{-2}\right) x_{h j} x_{i \ell} x_{m n} z_{\Psi} \\
= & q^{2}\left(q^{2}-q^{-2}\right)^{2} x_{h n} x_{i \ell} x_{m j} z_{\Psi}+q\left(q^{2}-q^{-2}\right) x_{i n} x_{h j} z_{\Psi} \\
& -q\left(q^{2}-q^{-2}\right) x_{h j} x_{i n} z_{\Psi}-\left(q^{2}-q^{-2}\right)^{2} x_{h n} x_{m j} x_{i \ell} z_{\Psi} \\
= & q\left(q^{2}-q^{-2}\right)^{2} x_{h n} x_{i j} z_{\Psi}-q\left(q^{2}-q^{-2}\right)^{2} x_{h n} x_{i j} z_{\Psi}=0 .
\end{aligned}
$$

Proof of Lemma 4.5. To define $f\left(x_{\lambda}, z_{\Sigma}\right)$ satisfying $(A)$ and $(B)$, we proceed by induction on $\eta(\lambda)+\eta(\Sigma)$. If $\eta(\lambda)+\eta(\Sigma)=1$, only the case $\lambda=(1,2)$ and $\Sigma$ $=(\phi)$ occurs; therefore we can put $f\left(x_{12}, 1\right)=z_{12}$. Assume that we have already defined the elements $f\left(x_{\lambda^{\prime}}, z_{\Sigma^{\prime}}\right) \in \mathfrak{S}_{q}(\mathcal{N})$ for $\lambda^{\prime}, \Sigma^{\prime}$ with $\eta\left(\lambda^{\prime}\right)+\eta\left(\Sigma^{\prime}\right)<\eta(\lambda)+\eta(\Sigma)$ so that they satisfy $(A)$ and $(B)$. We define $f\left(x_{\lambda}, z_{\Sigma}\right)$ when $\Sigma$ is increasing. For the case $\lambda \leq \Sigma$, we define $f\left(x_{\lambda}, z_{\Sigma}\right)=z_{\lambda} z_{\Sigma}$. If $\lambda \leq \Sigma$ fails, then $\Sigma=(\mu, T), \mu \leq T$ and $\mu<\lambda$. Put $(i, j)=\mu,(m, n)=\lambda$. By $(A)$ and (4.3 and 4$)$, we can put:

$$
f\left(x_{m n}, z_{\Sigma}\right)=\varepsilon_{i j m n} z_{i j} z_{m n} z_{T}+\varepsilon_{i j m n} f\left(x_{i j}, f\left(x_{m n}, z_{T}\right)-z_{m n} z_{T}\right)-X_{((i, j),(m, n))}
$$

where

$$
X_{((i, j),(m, n))}= \begin{cases}q f\left(x_{i n}, z_{T}\right) & \text { if }((i, j),(m, n)) \in C_{(\mathrm{V})} \\ \left(q^{2}-q^{-2}\right) f\left(x_{i n}, f\left(x_{m j}, z_{T}\right)\right) & \text { if }((i, j),(m, n)) \in C_{(\mathrm{IV})} \\ 0 & \text { if }((i, j), \\ (m, n)) \in C_{(\mathrm{I})} \cup C_{(\mathrm{II})} \cup C_{(\mathrm{III})} \cup C_{(\mathrm{VI})} .\end{cases}
$$

Since $\varepsilon_{i j m n} z_{i j} z_{m n} z_{T}=z_{m n} z_{\Sigma}, f\left(x_{m n}, z_{\Sigma}\right)$ satisfies $(B)$. By the definition of $f, f$ satisfies $(C)$ in the case $(i, j) \leq T$. We shall consider the case when $(i, j) \leq T$ fails. Write $T=((h, \ell), \Psi)$ where $(h, \ell) \leq \Psi,(h, \ell)<(i, j)$. By induction on $\eta(i, j)+\eta(m, n)+\eta(T)$, we show that $f$ satisfies $(C)$. Assume that, for each $\eta(i, j)$ $+\eta(m, n)+\eta(T) \leq r,(C)$ holds. Then, for $\eta(i, j)+\eta(m, n)+\eta(T) \leq r+1$, we have: 


$$
\begin{aligned}
x_{i j} & x_{m n} x_{h \ell} z_{\Psi} \\
& =x_{i j}\left(\varepsilon_{h \ell m n} x_{h \ell} x_{m n} z_{\Psi}-y_{h \ell m n} z_{\Psi}\right) \\
& =\varepsilon_{h \ell m n}\left(\varepsilon_{h \ell i j} x_{h \ell} x_{i j} x_{m n} z_{\Psi}-y_{h \ell i j} x_{m n} z_{\Psi}\right)-x_{i j} y_{h \ell m n} z_{\Psi} \\
& =\varepsilon_{h \ell m n} \varepsilon_{h \ell i j} x_{h \ell} x_{i j} x_{m n} z_{\Psi}-\varepsilon_{h \ell m n} y_{h \ell i j} x_{m n} z_{\Psi}-x_{i j} y_{h \ell m n} z_{\Psi}
\end{aligned}
$$

(Here we abbreviate $f\left(x_{i_{1}, j_{1}}, f\left(x_{i_{2}, j_{2}}, \ldots, f\left(x_{i_{t}, j_{t}}, z_{\Psi}\right).\right)\right.$ ) to $\left.x_{i_{1}, j_{1}} x_{i_{2}, j_{2}} \cdots x_{i_{t}, j_{t}} z_{\Psi}.\right)$ Similary,

$$
\begin{aligned}
& x_{m n} x_{i j} x_{h \ell} z_{\Psi} \\
&= \varepsilon_{h \ell i j} \varepsilon_{h \ell m n} \varepsilon_{i j m n} x_{h \ell} x_{i j} x_{m n} z_{\Psi}-\varepsilon_{h \ell i j} \varepsilon_{h \ell m n} x_{h \ell} y_{i j m n} z_{\Psi} \\
& \quad-\varepsilon_{h \ell i j} y_{h \ell m n} x_{i j} z_{\Psi}-x_{m n} y_{h \ell i j} z_{\Psi} .
\end{aligned}
$$

Therefore, by Lemma 4.6, we get:

$$
\varepsilon_{i j m n} x_{i j} x_{m n} z_{T}-x_{m n} x_{i j} z_{T}-y_{i j m n} z_{T}=0 .
$$

Hence $f$ satisfies $(C)$. This completes the proof.

We can restate Lemma 4.5 as:

Lemma 4.7. $\mathfrak{S}_{q}(\mathcal{N})$ has a left $U_{q}(\mathcal{N})$-module structure satisfying:

(A) $\tilde{x}_{\lambda} z_{\Sigma}=z_{\lambda} z_{\Sigma} \quad$ for $\lambda \leq \Sigma$.

(B) $\quad \tilde{x}_{\lambda} z_{\Sigma}=z_{\lambda} z_{\Sigma} \quad\left(\bmod S_{\eta(\Sigma)+\eta(\lambda)-1}\right)$.

As in the proof of Lemma 2.2, Lemma 4.2 and Lemma 4.7 imply the following:

Lemma 4.8. Let $q \in F^{\times}$be such that $q^{8} \neq 1$. Then the set $\left\{z_{\Sigma} \mid \sum\right.$ increasing $\}$ is a basis of $U_{q}(\mathscr{N})$.

Combining Proposition 2.3, and Lemma 4.1 with Lemma 4.8, we obtain Theorem 1.1.

\section{\$5. Proof of Theorem 1.2}

Let $\Sigma=\left(\left(i_{1}, j_{1}\right), \ldots,\left(i_{t}, j_{t}\right)\right) \in P_{N}$. Define the function $\delta: P_{N} \rightarrow \mathbb{Z}$ by $\delta(\Sigma)$ $=\left(j_{1}-i_{1}\right)+\cdots+\left(j_{t}-i_{t}\right)$ and $\delta((\phi))=0$. Denote the element $e_{i_{1}, j_{1}} \cdots e_{i_{t}, j_{t}}$ (resp. $\left.f_{i_{1}, j_{1}} \cdots f_{i_{t}, j_{t}}\right)$ of $U_{q}\left(s l_{N+1}(F)\right)$ by $e_{\Sigma}\left(\right.$ resp. $\left.f_{\Sigma}\right)$. We understand $e_{(\phi)}=f_{(\phi)}=1$. Put

$$
\Omega_{N}=\left\{(m, n) \in \mathbb{Z} \times \mathbb{Z} \mid m, n \geq 0, n \leq \mathscr{M} a x\left\{\eta(\Sigma) \mid \Sigma \in P_{N}, \delta(\Sigma)=m\right\}\right\} .
$$

For $(m, n) \in \Omega_{N}$, let $\mathfrak{A}_{(m, n)}$ be the subspace of $U_{q}\left(s l_{N+1}(F)\right)$ spanned by the elements $f_{\Sigma^{\prime}} k_{1}^{\ell}{ }^{1} \cdots k_{N}^{\ell} e_{\Sigma}$, where $\Sigma$ and $\Sigma^{\prime}$ are increasing, $\left(\delta\left(\Sigma^{\prime}\right)+\delta(\Sigma), \eta\left(\Sigma^{\prime}\right)\right.$ $+\eta(\Sigma)) \leq(m, n)$, and $\ell_{1}, \ldots, \ell_{N} \in \mathbb{Z} . \quad$ Clearly, $\mathfrak{U}_{\left(m^{\prime}, n^{\prime}\right)} \mathfrak{A}_{(m, n)} \subset \mathfrak{A}_{\left(m^{\prime}+m, n^{\prime}+n\right)}, \mathfrak{U}_{(m, n)}$ 
$\subset \mathfrak{A}_{(i, j)}$ if $(m, n)<(i, j)$. For $(m, n) \in \Omega_{N}$, let $(m, n)^{\wedge} \in \Omega_{N}$, be the element satisfying that

(1) $(m, n)^{\wedge}<(m, n)$.

(2) There is no element $(i, j) \in \Omega_{N}$ such that $(m, n)^{\wedge}<(i, j)<(m, n)$.

Put $\overline{\mathfrak{A}}_{(m, n)}=\mathfrak{\mathscr { A }}_{(m, n)} / \mathfrak{U}_{(m, n)} \wedge$, and $\overline{\mathfrak{A}}_{(0,0)}=\mathfrak{\mathfrak { U }}_{(0,0)}$. Then $\overline{\mathfrak{A}}_{N}=\underset{(m, n) \in \Omega_{N}}{\bigoplus} \overline{\mathfrak{A}}_{(m, n)^{\wedge}}$. has a graded algebra structure with 1 whose multiplication is defined component-wise by

$$
\left(x+\mathfrak{U}_{(m, n)^{\wedge}}\right)\left(y+\mathfrak{U}_{\left(m^{\prime}, n^{\prime}\right)^{\wedge}}\right)=x y+\mathfrak{U}_{\left(m+m^{\prime}, n+n^{\prime}\right)^{\wedge}},
$$

where $x \in \mathfrak{A}_{(m, n)}, y \in \mathfrak{A}_{\left(m^{\prime}, n^{\prime}\right)}$. Let $\mathfrak{S}_{q}(\mathscr{G})$ be the associative $F$-algebra with 1 with generators $\tilde{e}_{i j}, \tilde{f}_{i j}\left((i, j) \in \Lambda_{N}\right), \tilde{k}_{i}^{\mp 1},(1 \leq i \leq N)$ and relations:

$$
\begin{aligned}
& \tilde{k}_{i} \tilde{k}_{i}^{-1}=\tilde{k}_{i}^{-1} \tilde{k}_{i}=1, \tilde{k}_{i} \tilde{k}_{j}=\tilde{k}_{j} \tilde{k}_{i} \\
& \tilde{k}_{r} \tilde{e}_{i j} \tilde{k}_{r}^{-1}=q^{\left(a_{1, r}+a_{i+1, r}+\cdots+a_{j-1, r}\right)} \tilde{e}_{i j} \\
& \tilde{k}_{r} \tilde{f}_{i j} \tilde{k}_{r}^{-1}=q^{-\left(a_{i, r}+a_{i+1, r}+\cdots+a_{j-1, r}\right)} \tilde{f}_{i j} \\
& \tilde{e}_{i j} \tilde{f}_{m n}=\tilde{f}_{m n} \tilde{e}_{i j} \\
& \left.\varepsilon_{i j m n} \tilde{e}_{i j} \tilde{e}_{m n}=\tilde{e}_{m n} \tilde{e}_{i j}, \varepsilon_{i j m n} \tilde{f}_{i j} \tilde{f}_{m n}=\tilde{f}_{m n} \tilde{f}_{i j}((i, j)<m, n)\right) .
\end{aligned}
$$

As in the proof of Lemma 2.2, we can show that the elements $\tilde{f}_{m_{1}, n_{1}} \cdots \tilde{f}_{m_{S}, n_{s}} \tilde{k}_{1}^{\ell} \cdots \tilde{k}_{N}^{\ell N} \tilde{e}_{i_{1}, j_{1}} \cdots \tilde{e}_{i_{t}, j_{t}}\left(\left(m_{1}, n_{1}\right) \leq \cdots \leq\left(m_{s}, n_{s}\right), \quad\left(i_{1}, j_{1}\right) \leq \cdots \leq\left(i_{t}, j_{t}\right)\right.$, $\left.\ell_{1}, \ldots, \ell_{N} \in \mathbb{Z}\right)$ form an $F$-basis of $\mathfrak{S}_{q}(\mathscr{G})$. As an immediate consequence of Theorem 1.1 and the formulas in $\S 3$, we get:

Lemma 5.1. $\overline{\mathfrak{A}}_{N}$ is isomorphic to $\mathfrak{S}_{q}(\mathscr{G})$ as F-algebras.

Remark. The above argument shows that, using our filtration, we can compute the structure constants of $U_{q}\left(s l_{N+1}(F)\right)$ with respect to the basis given in Theorem 1.1.

Lemma 5.2. (a) $\mathfrak{S}_{q}(\mathscr{G})$ has no zero divisors $\neq 0$.

(b) $\mathfrak{S}_{q}(\mathscr{G})$ is a left (right) Noetherian ring.

Since $\mathfrak{S}_{q}(\mathscr{G})$ is a non-commutative analogue of polynomial rings, $(a)$ and $(b)$ can be proved in a way similar to the case of usual polynomial ring (e.g. see [10, Th. 1.2.10]).

By Lemma 5.1 and Lemma 5.2, we obtain Theorem 1.2. The proof is entirely similar to that of [5, Chap. 4 , Theorem 4$]$. 


\section{§6. $\mathbb{U}_{q}\left(s l_{N+1}(\mathbb{R})\right)$ over a Commutative $\mathbb{R}$ ing $\mathbb{R}$}

Let $R$ be a commutative ring with 1 , and $R^{\times}$the unit group of $R$. Assume $q \in R^{\times}$is such that $q^{4}-1 \in R^{\times}$. Let $U_{q}\left(s l_{N+1}(R)\right)$ be the associative $R$-algebra with 1 with generators $e_{i}, f_{i}, k_{i}^{ \pm 1}(1 \leq i \leq N)$, and relations (1.1), .., (1.5), where $A=\left(a_{i j}\right)_{1 \leq i, j \leq N}$ is the Cartan matrix of type $A_{N}$. For $1 \leq i<j \leq N+1$, we define $e_{i j}, f_{i j}$ by (1.6). Let $L$ be the two sided ideal of $U_{q}\left(s l_{N+1}(R)\right)$ generated by $\left[e_{i, i+3}, e_{i+1}\right],\left[f_{i, i+3}, f_{i+1}\right](1 \leq i \leq N-2)$. Put $\bar{U}_{q}\left(s l_{N+1}(R)\right)=U_{q}\left(s l_{N+1}(R)\right) / L$. By (3.3), for a field $F$, if $q^{8} \neq 1, \bar{U}_{q}\left(s l_{N+1}(F)\right) \simeq U_{q}\left(s l_{N+1}(F)\right) / L$.

Theorem 6.1. (a) As an R-module, $\bar{U}_{q}\left(s l_{N+1}(R)\right)$ is free. The elements

$$
f_{m_{1}, n_{1}} \cdots f_{m_{s}, n_{s}} k_{1}^{\ell} \cdots k_{N}^{\ell N} e_{i_{1}, j_{1}} \cdots e_{i_{t}, j_{t}}
$$

$\left(\left(m_{1}, n_{1}\right) \leq \cdots \leq\left(m_{s}, n_{s}\right),\left(i_{1}, j_{1}\right) \leq \cdots \leq\left(i_{t}, j_{t}\right), \ell_{1}, \ldots, \ell_{N} \in \mathbb{Z}\right)$ form an $R$-basis of $\bar{U}_{q}\left(s l_{N+1}(R)\right)$.

(b) If $R$ has no zero divisors $\neq 0$, then $\bar{U}_{q}\left(s l_{N+1}(R)\right)$ has no zero divisors $\neq 0$. If $R$ is a Noetherian ring, $\bar{U}_{q}\left(s l_{N+1}(R)\right)$ is a left (right) Noetherian ring.

(c) If $q^{8}-1 \in R^{\times}$, then $L=(0)$.

Proof. First we prove $(a)$. Let $v$ be an indeterminate, $\mathbb{C}(v)$ the field of rational functions. Let $\mathscr{A}$ be the $\mathbb{Z}$-subalgebra of $\mathbb{C}(v)$ generated by $1, v^{\mp 1},\left(v^{4}\right.$ $-1)^{-1}$. We define $U_{\mathscr{A}}$ to be the $\mathscr{A}$-submodule of $U_{v}\left(s l_{N+1}(\mathbb{C}(v))\right.$ generated by the elements (6.1). From the arguments in $\S 5$, we see that $U_{\mathscr{A}}$ is an $\mathscr{A}$-algebra with a free $\mathscr{A}$-basis (6.1). We now define, for $R$ and $q, U_{R, q}=U_{\mathscr{A}} \otimes \mathscr{A}_{q}$, where $R_{q}$ is $R$, regarded as an $\mathscr{A}$-algebra with $v$ (resp. 1) acting as multiplication by $q$ (resp. 1). Since $U_{R, q}$ satisfies the formulas $\left[e_{i, i+3}, e_{i+1}\right]=\left[f_{i, i+3}, f_{i+1}\right]=0$, we can define the epimorphism $\varphi: \bar{U}_{q}\left(s l_{N+1}(R)\right) \rightarrow U_{R, q}$ by $\varphi\left(e_{i}\right)=e_{i} \otimes 1, \varphi\left(f_{i}\right)$ $=f_{i} \otimes 1, \varphi\left(k_{i}^{\mp 1}\right)=k_{i}^{\mp 1} \otimes 1$. Hence the elements (6.1) are linearly independence over $R$. We know that the elements $e_{i j}, f_{i j}$ and $k_{i}$ of $\bar{U}_{q}\left(s l_{N+1}(R)\right)$ satisfies the formulas (1), (2), (3), (4) in $\S 3$. Hence, defining the filtration on $\bar{U}_{q}\left(s l_{N+1}(R)\right)$ similar to $\left\{\mathfrak{H}_{(m, n)}\right\}_{(m, n) \in \Omega_{N}}$ in $\S 5$, we see that, as an $R$-module, $\bar{U}_{q}\left(s l_{N+1}(R)\right)$ is generated by the elements (6.1). This completes the proof of $(a)$.

We obtain $(b)$ from the same argument as in $\$ 5$, and $(c)$ from the formula (3.3).

For $U_{q}\left(s l_{N+1}(F)\right)$ over a field $F$, we have the following supplementary result:

Proposition 6.2. Assume $q \in F^{\times}$is a primitive 8-th root of unity. Then the elements

$$
\begin{gathered}
f_{m_{1}, n_{1}} f_{m_{2}, n_{2}} \cdots f_{m_{s}, n_{s}} f_{1}^{2 b_{1}} \cdots f_{N}^{2 b_{N}} k_{1}^{\ell}{ }^{\ell} \cdots k_{N}^{\ell N} e_{i_{1}, j_{1}} e_{i_{2}, j_{2}} \cdots e_{i_{t}, j_{t}} e_{1}^{2 c_{1}} \cdots e_{N}^{2 c_{N}} \\
\left(m_{p}<n_{p+1}-1(1 \leq p \leq s-1), i_{r}<j_{r+1}-1(1 \leq r \leq t-1),\right. \\
\left.b_{1}, \ldots, b_{N}, c_{1}, \ldots, c_{N} \geq 0, \ell_{1}, \ldots, \ell_{N} \in \mathbb{Z}\right)
\end{gathered}
$$


form a basis of $U_{q}\left(s l_{N+1}(F)\right)$.

The proof is based on Proposition 2.3 and an argument similar to the one used in the proof of Lemma 2.1,2.2; we omit the details.

From Proposition 6.2, we obtain:

Proposition 6.3. Assume $q$ to be an indeterminate. Let $\mathscr{A}=F\left[q^{ \pm 1},\left(q^{4}\right.\right.$ $\left.-1)^{-1}\right]$. Then, if $N \geq 3, U_{q}\left(s l_{N+1}(\mathscr{A})\right)$ is not free as an $\mathscr{A}$-module.

Proof. By the argument of (3.3), we see

$$
\left(q^{2}+q^{-2}\right)\left[e_{i, i+3}, e_{i+1, i+2}\right]=0 .
$$

Let $\zeta \in F$ be a primitive 8-th root of unity. Define the $F$-algebra homomorphism $p: U_{q}\left(s l_{N+1}(\mathscr{A})\right) \rightarrow U_{\zeta}\left(s l_{N+1}(F)\right)$ by $p\left(e_{i}\right)=e_{i}^{\prime}, p\left(f_{i}\right)=f_{i}^{\prime}, p\left(k_{i}^{ \pm 1}\right)=k_{i}^{\prime \pm 1}, p(q)$ $=\zeta$ where $e_{i}^{\prime}, f_{i}^{\prime}, k_{i}^{\prime \prime 1}(1 \leq i \leq N)$ are generators in the definition of $U_{\zeta}\left(s l_{N+1}(F)\right)$. By Proposition 6.2, we have

$$
p\left(\left[e_{i, i+3}, e_{i+1, i+2}\right]\right)=e_{i, i+3}^{\prime} e_{i+1, i+2}^{\prime}-e_{i+1, i+2}^{\prime} e_{i, i+3}^{\prime} \neq 0 .
$$

Hence $\left[e_{i, i+3}, e_{i+1, i+2}\right] \neq 0$, which shows that $U_{q}\left(s l_{N+1}(\mathscr{A})\right)$ is not free.

\section{§7. On $U_{q}\left(s o_{5}(F)\right)$}

Let $A$ be the Cartan matrix of type $B_{2}$, namely, $A=\left(\begin{array}{cc}2 & -2 \\ -1 & 2\end{array}\right)$. Put $U_{q}\left(\operatorname{so}_{5}(F)\right)=U_{q}(A, \operatorname{diag}(1,2))$ where $q^{8} \neq 1$. Define the elements $E_{i}, F_{i}(1 \leq N)$ by

$$
\begin{aligned}
& E_{1}=e_{1}, E_{2}=e_{2}, E_{3}=e_{1} e_{2}-q^{4} e_{2} e_{1}, E_{4}=e_{1} E_{3}-q^{-4} E_{3} e_{1}, \\
& F_{1}=f_{1}, F_{2}=f_{2}, F_{3}=f_{1} f_{2}-q^{4} f_{2} f_{1}, F_{4}=f_{1} F_{3}-q^{-4} F_{3} f_{1} .
\end{aligned}
$$

Proposition 7.1. (a) The elements $F_{1}^{m_{1}} F_{2}^{m_{2}} F_{3}^{m_{3}} F_{4}^{m_{4}} \cdot k_{1}^{\ell_{1}} k_{2}^{\ell_{2}} \cdot E_{1}^{i_{1}} E_{2}^{i_{2}} E_{3}^{i_{3}} E_{4}^{i_{4}}$ $\left(m_{s}, i_{s} \geq 0, \ell_{1}, \ell_{2} \in \mathbb{Z}\right)$ form a basis of $U_{q}\left(\operatorname{so}_{5}(F)\right)$.

(b) $U_{q}\left(\mathrm{so}_{5}(F)\right)$ is a left (right) Noetherian ring, and has no zero divisors $\neq 0$.

Let $V_{i}$ be the subspace of $U_{q}\left(s_{5}(F)\right)$ spanned by the elements $F_{1}^{m_{1}} F_{2}^{m_{2}} F_{3}^{m_{3}} F_{4}^{m_{4}} \cdot k_{1}^{\ell_{1}} k_{2}^{\ell 2} \cdot E_{1}^{i_{1}} E_{2}^{i_{2}} E_{3}^{i_{3}} E_{4}^{i_{4}}$ such that $2\left(m_{1}+i_{1}\right)+\left(m_{2}+i_{2}\right)+2\left(m_{3}\right.$ $\left.+i_{3}\right)+3\left(m_{4}+i_{4}\right) \leq i$. The proofs of $(a)$ and $(b)$ are obtained by using the filtration $\left\{V_{i}\right\}_{i=0}^{\infty}$ of $U_{q}\left(s_{5}(F)\right)$. This is similar to those of Theorem 1.1 and 1.2. The details are omitted.

\section{Acknowledgements.}

The author would like to express his heartfelt thanks to Professor M. Jimbo for showing him some useful formulas which were necessary in proving our 
theorem, and to Professor A. Gyoja for pointing out that the Noetherian property of $U_{q}\left(s l_{N+1}(F)\right)$ follows easily from its filtration. He also thanks $\mathrm{Y}$. Izumi for some very interesting discussion.

\section{References}

[1] Bourbaki, N., Groupes et Algèbres de Lie, Chaps. I, Hermann, Paris, 1960.

[2] Drinfeld, V. G., Hopf algebra and the quantum Yang-Baxter equation, Sov. Math. Dolk., 32 (1985), 254-258.

[3] - Quantum groups, (Proc. I. C. M. Berkerey, 1986).

[4] Izumi, Y., On a $q$-analogue of the universal enveloping algebra of the simple Lie algebra of type $A_{N}$, Master thesis, Osaka University (1988) (in Japanese).

[5] Jacobson, N, Lie Algebras, Interscience, New York, 1962.

[6] Jimbo, M., A q-difference analogue of $U(\mathscr{G})$ and the Yang-Baxter equation, Lett. Math. Phys., 10 (1985), 63-69.

[7] - A $q$-analogue of $U(g l(N+1))$, Hecke algebra, and the Yang-Baxter equation, Lett. Math. Phys., 11 (1986), 247-252.

[8] Kac, V., G., Infinite dimensional Lie algebra, Progress in Mathematics, 44, Birkhäuser, Boston/Basel/Stuttgart, 1983.

[9] Lusztig, G., Quantum deformations of certain simple modules over enveloping algebra, Advances in Math., 70 (1988), 237-249.

[10] McConnell, J. C., and Robson, J. C., Noncommutative Noetherian rings, Wiley-Interscience, Chichester/New York/Brisbane, 1988.

[11] Rosso, M., Finite dimensional representations of the quantum analog of the enveloping algebra of a complex simple Lie algebra, Commun. Math. Phys., 117 (1988), 581-593. 